\title{
Reflets
}

Revue ontaroise d'intervention sociale et communautaire

\section{Perceptions de la criminalité et évaluation du niveau de sécurité : une étude qualitative dans un quartier sudburois}

\section{Viviane Munroe}

Volume 7, numéro 2, automne 2001

Le travail social en Ontario

URI : https://id.erudit.org/iderudit/026363ar

DOI : https://doi.org/10.7202/026363ar

Aller au sommaire du numéro

Éditeur(s)

Reflets : Revue ontaroise d'intervention sociale et communautaire

ISSN

1203-4576 (imprimé)

1712-8498 (numérique)

Découvrir la revue

Citer cet article

Munroe, V. (2001). Perceptions de la criminalité et évaluation du niveau de sécurité : une étude qualitative dans un quartier sudburois. Reflets, 7(2),

151-156. https://doi.org/10.7202/026363ar

Tous droits réservés (C) Reflets : Revue ontaroise d'intervention sociale et communautaire, 2001
Ce document est protégé par la loi sur le droit d'auteur. L'utilisation des services d'Érudit (y compris la reproduction) est assujettie à sa politique d'utilisation que vous pouvez consulter en ligne.

https://apropos.erudit.org/fr/usagers/politique-dutilisation/ 


\section{Perceptions de la criminalité et évaluation du niveau de sécurité : une étude qualitative dans un quartier sudburois}

par

Viviane Munroe,

étudiante, École de service social, Université Laurentienne

\section{Introduction}

L'Association Cabot Park Kids organise des activités pour les résidents des logements subventionnés qui occupent près d'une centaine de logis sur les rues Cabot, Hearne et Burton à Sudbury. Lors de sa réunion annuelle, à l'automne 2000, il est venu à l'attention du comité de parents que la criminalité juvénile semblait être un problème grandissant dans le quartier. L'opinion dominante était que la délinquance constituait un problème réel et inquiétant qui provoquait une multitude de sentiments tels l'insécurité et la méfiance envers les jeunes. À partir de cette opinion, nous avons élaboré un plan de recherche qui a permis d'explorer et de décrire comment les résidents du quartier perçoivent la criminalité juvénile et comment ils évaluent le niveau de sécurité de leur quartier. En menant des entrevues auprès de cette population, nous avons tenté de comprendre les perceptions du problème et si celles-ci étaient assez sérieuses pour entreprendre des actions. 


\section{Méthodologie}

L'étude a été menée auprès des résidents de Cabot Park, c'est-àdire dans un milieu résidentiel de logements subventionnés comprenant 87 logis et s'étendant sur les rues Cabot, Hearne et Burton de Sudbury. Les sujets qui ont participé à cette étude étaient des individus âgés de 18 ans et plus qui demeuraient dans le quartier. L'échantillon de cette recherche a été constitué à partir de la technique «boule de neige». Un questionnaire a été l'instrument de collecte des données de cette étude. Il était composé de deux parties. La première partie était constituée de huit questions ouvertes, traitées de façon qualitative, faisant appel aux perceptions et aux sentiments du sujet par rapport à la délinquance et à la sécurité du quartier. La deuxième partie était composée de huit questions fermées, qui ont fait l'objet d'un traitement quantitatif et descriptif.

\section{Description de l'échantillon}

L'échantillon était constitué de 20 individus âgés de 23 à 45 ans. L'âge moyen de l'échantillon était 33,4 ans. Le sexe masculin était sous-représenté, $75 \%$ de l'échantillon étant du sexe féminin et $35 \%$ du sexe masculin. Quatre-vingt-dix pour cent de l'échantillon dispose d'un revenu de moins de $25,000 \$$, ce qui peut apparaitre comme un reflet du niveau de scolarisation des personnes interrogées dont $50 \%$ possède uniquement un diplôme d'études secondaires alors qu'aucune personne n'a de formation universitaire et que trois ont une formation professionnelle.

\section{Analyse et résultats}

Dans un premier temps, on a demandé aux informateurs 
d'indiquer quels problèmes étaient présents dans le quartier. L'intention était de constater, selon les réponses des informateurs, si la délinquance était perçue comme un problème. Pour ce faire, on a observé la fréquence de l'usage du terme «délinquance» et des thèmes reliés à ce concept dans le discours des informateurs. Par la suite, pour comprendre comment ils percevaient la délinquance, on les a interrogés sur quatre aspects particuliers: le taux de délinquance, le temps de l'année où elle se manifeste, les facteurs qui y contribuent ainsi que des stratégies pour la prévenir. Pour l'évaluation de la sécurité du quartier, on a utilisé quatre variables: on a demandé aux sujets s'ils ressentaient un besoin de sécurité dans le quartier, s'ils pensaient que le quartier était sécuritaire la nuit, ce qu'ils pensaient du niveau de surveillance par la police et s'ils pensaient que le taux de criminalité juvénile était plus élevé dans ce quartier qu'ailleurs.

Bien que les divers problèmes énumérés et décrits par l'échantillon ne se limitent pas aux jeunes, un grand nombre de répondants en ont parlé comme étant lié aux jeunes. L'ensemble de ces problèmes a été analysé et trois grandes catégories en découlent:la délinquance, le désœuvrement et le manque de bienveillance. La catégorie «délinquance» a été utilisée pour signifier tous les actes commis par des jeunes moins de 18 ans qui enfreignent la loi. Parmi les actes reliés à cette catégorie, le vol et le vandalisme ont été rapportés le plus souvent par les répondants. La violence,l'alcoolisme et les bagarres ont aussi été mentionnés par l'échantillon, mais ces actes et comportements semblaient être moins fréquents. Sous le concept de «désœuvrement», on a rassemblé tous les énoncés qui indiquaient que les jeunes n'avaient rien à faire pour occuper leur temps à des activités productives et positives. Un grand nombre de répondants a indiqué que ceci est un problème non seulement parce que les jeunes dérangent la vie privée en passant dans les cours d'autrui et en faisant du bruit tard la nuit, mais aussi parce que l'oisiveté mène à la délinquance. Le «manque de bienveillance» est une autre catégorie créée pour inclure tous les actes qui vont contre la morale et les valeurs sociales. Bien que ce problème ne soit pas le plus important, il a été soulevé sous la forme d'un manque de respect, de l'usage de langage vulgaire et de racisme chez les jeunes. 
Les résultats ont démontré que la majorité des informateurs perçoivent la criminalité juvénile comme un problème. Près de la moitié des personnes interrogées a affirmé que le taux de délinquance est vraiment élevé et un autre tiers a tout simplement dit qu'il était élevé. Ce problème se manifeste surtout l'été, quand les jeunes n'ont pas d'activités pour se tenir occupés. D'après l'échantillon, ce problème est particulièrement dû au manque de supervision et de discipline de la part des parents. D'autres causes seraient la pauvreté, le manque d'activités pour les jeunes et les troubles familiaux. Les répondants avaient aussi à exprimer des stratégies pour prévenir la délinquance. Parmi celles-ci, on trouvait la création d'activités pour occuper les jeunes, la mise en place de programmes pour surveiller le quartier et l'organisation de sessions d'informations ou de counselling pour les jeunes et leurs parents. D'autres étaient d'avis que le problème de la délinquance ne pouvait être réglé que par la modification des lois portant sur les jeunes en les rendant plus sévères.

Quatre variables portaient sur l'évaluation de la sécurité du quartier, sans compter la variable concernant le statut de victime. Ces variables indiquent que $80 \%$ des répondants avaient été victimes d'un crime commis par un jeune moins de 18 ans. D'après les données, on constate que $75 \%$ des répondants a affirmé que le quartier n'était pas sécuritaire la nuit. Seulement deux informateurs ont affirmé ne pas ressentir un tel sentiment. Toutefois, cette question était quantitative et les informateurs ne pouvaient pas mettre leur réponse en contexte. Nous avons observé, lors des entrevues, que chaque répondant a hésité lorsque le temps est venu d'encercler la réponse à cette question. L'un des sujets a encerclé les deux énoncés, car il était ambivalent par rapport à son sentiment. Parmi les deux répondants ayant indiqué qu'ils se sentaient en sécurité la nuit, l'un a expliqué qu'il répondait ainsi parce qu'il ne connaissait pas suffisamment le quartier et l'autre affichait une faible conviction. En ce qui concerne le niveau de surveillance de la police, plus de la moitié des répondants jugeait qu'il n'était pas suffisamment élevé. Il y avait deux commentaires qui revenaient souvent. Le premier est à l'effet que la police ne surveille pas vraiment et que sa présence dans le quartier 
correspond à une destination précise pour y faire une arrestation. Le second est à l'effet que la police n'agit pas assez rapidement lorsqu'elle est appelée. En comparant leur quartier à d'autres quartiers sudburois, un peu plus de la moitié des répondants a affirmé que le niveau de délinquance n'était pas plus élevé qu'ailleurs. Les résultats précédents semblent indiquer que les répondants ressentent de l'insécurité dans le quartier. Toutefois, en leur demandant de comparer ce quartier avec d'autres, on se rend compte que les répondants ne se sentent pas plus en danger dans ce quartier que dans d'autres quartiers de logements subventionnés sudburois.

\section{Discussion et conclusion}

Dans l'ensemble, les résultats correspondent aux affirmations et aux opinions émises lors de la réunion de l'Association Cabot Park Kids de l'automne 2000. La délinquance est perçue comme un problème dans le quartier. Il est clair que la relation entre les jeunes et les adultes du quartier pourrait s'améliorer. De même, les causes de la délinquance et les stratégies identifiées pour la prévenir présentent plusieurs similitudes à celles soulevées lors de la réunion d'automne. Toutefois, nous n'avons pas exploré le concept d'insécurité en profondeur. Nous ne pouvons donc pas discuter des diverses formes de manifestation de l'insécurité. Toutefois, les gens ont parlé de vols et de vandalisme. Ainsi, l'insécurité évoquée serait davantage reliée au risque de se faire prendre ou endommager un bien difficilement remplaçable en raison de la condition économique des répondants qu'à une insécurité relevant des crimes contre la personne comme les voies de fait.

Cette recherche a permis de décrire comment les gens du quartier perçoivent la délinquance, mais il a été plus difficile de comprendre comment ils évaluent la sécurité. Conformément aux recherches antérieures, cette étude montre la complexité de la recherche sur les perceptions de la criminalité et sur les 
évaluations de la sécurité. Peu importe les types d'études, il y a tellement d'aspects qui entrent en jeu lorsqu'il s'agit de faire l'étude de ces phénomènes, qu'il est difficile de tous les intégrer. On a remarqué qu'une personne pouvait penser que le taux de criminalité est élevé dans son quartier, sans que cela signifie qu'elle a peur ou qu'elle ressent de l'insécurité. D'autres sentiments tels la colère et la méfiance envers les jeunes, peuvent également influencer la perception d'un niveau élevé de criminalité juvénile dans un quartier.

\section{Bibliographie}

BABA, Y. et D. MARTIN AUSTIN (1989). «Neighborhood Environmental Satisfaction,Victimization, and Social Participation as Determinants of Perceived Neighborhood Safety», Environment and Behaviour, November, 763-781.

HOLZMAN, Harold R. (1996). "Criminological Research on Public Housing: Towards a Better Understanding of People, Places, and Spaces», Crime and Delinquency, July, vol. 42, 361-377.

MCDOWALL, David, Colin LOFTIN et Brian WIERSEMA (2000). «The Impact of Youth Curfew Laws on Juvenile Crime Rates», Crime and Delinquency, January, vol. 46, no 1,77-91.

MC QUAIL, Denis (1983). Mass Communication Theory, An Introduction, London, J.W.Arrosmith Ltd.

SACCO,Vincent F. (1982). «The Effects of Mass Media on the Perception of Crime: a Reanalysis of the issues», Pacific sociological Review, vol. 25, no. 4, October, 475-493.

WILCOX ROUNTREE, Pamela (1998). "A Reexamination of the Crime-Fear Linkage», Journal of Research in Crime and Delinquency, vol. 35,341-371. 